Ocula ${ }^{16}$

Semiotics of Economic Discourse

Semiotica del discorso economico

a cura di Giorgio Coratelli, Francesco Galofaro, Federico Montanari

www.ocula.it | Dicembre 2015 - DOI: 10.12977/ocula42

\title{
Controtendenza del retail nella crisi del nuovo millennio
}

\section{Tiziana Barone}

tiziana_barone@alice.it

\begin{abstract}
L'economia al pari della lingua e della parentela è un vero e proprio sistema nel quale le relazioni tra le varie componenti costituiscono e trasformano le componenti stesse. Queste relazioni sistematiche sono di natura narrativa in quanto, i soggetti che fanno parte di questo sistema, costruiscono, decostruiscono e ricostruiscono la propria identità in funzione di altri soggetti con cui entrano in relazione ora contrattuale ora polemica.

In questo sistema di significazione interagiscono le due diverse forme dell'esperienza umana: la dimensione sensibile (la prassi economico-produttiva) e la parte intellegibile (valori economici e della marca). Il progredire della crisi economica negli ultimi anni ha fatto sì che in un ambito fondamentale della dimensione economica come il retail, si innestasse una particolare controtendenza. Partendo dalla narratività come principio esplicativo, la crisi può essere letta come un antisoggetto che entra in relazione polemica col soggetto impresa: questa implementerà delle tattiche che consentiranno di disgiungersi dall'attante oggetto, ovvero la chiusura dell'azienda. Alcune di queste tattiche prevedono la chiusura di punti vendita e in generale l'abbattimento dei costi sfruttando al massimo il web e le campagne social. Il contesto economico e le esperienze soggettive si modificano, per cui la dialettica fra ricezione e trasmissione in ciò che viene chiamato tendenza varia. La tendenza individua un microuniverso emergente "capace di far convergere verso di sé atteggiamenti e comportamenti” (Ceriani, 2007) che si estendono trasversalmente; una continua tensione tra valori preesistenti e usi della società, i quali perseguono lo stesso ritmo di mutazione. Ecco che si installa una concezione opposta alla tendenza a ridurre i costi, ovvero una controtendenza; dal punto di vista economico si fanno degli investimenti in retail da parte di aziende che nascono come brand on-line.
\end{abstract}

\section{Parole chiave}

Crisi economica; Tendenza; Controtendenza; Pop up store; Marketing

\section{Keywords}

Economic Crisis; Trends; Counter-tendencies; Pop-up stores; Marketing

\section{Sommario}

1. Significazione e ripresa economica viaggiano sullo stesso binario?

2. Sua maestà la finanza

3. Controtendenze: dall'on-line all'off-line

4. Una spazialità differente

5. Il temporay store

6. Definizioni e confusioni

7. Google 


\section{Ocula ${ }^{16}$}

Semiotics of Economic Discourse | Semiotica del discorso economico

Tiziana Barone| Controtendenza del retail nella crisi del nuovo millennio

8. Ebay

9. Conclusioni

Bibliografia

Pretendo di vivere pienamente la contraddizione del mio tempo, che di un sarcasmo può fare la condizione della verità.

Roland Barthes, Miti d'oggi

\section{Significazione e ripresa economica viaggiano sullo stesso binario?}

Nel 2007 ha origine la Grande Recessione, una crisi economica mondiale che inizia negli Stati Uniti con lo scoppio della bolla immobiliare, seguita dall'instabilità finanziaria. La recessione ha gradualmente assunto poi un carattere globale e perdurante (tranne alcune eccezioni, come Cina e India).

Il crollo del mercato subprime ${ }^{1}$ ha fatto sì che le società di prestiti abbiano pensato di cartolarizzare questi crediti, così come quelli verso la clientela solvibile, cioè a incorporare in un titolo cedibile sul mercato i diritti derivanti da tale credito così come fanno le banche ordinarie. Cartolarizzando i crediti, le banche ottengono nuovi capitali prontamente disponibili all'impiego e, nello stesso tempo, riducono in bilancio il livello dei crediti a rischio, in modo da ottenere un migliore rating.

Tra i principali fattori della crisi figurano gli alti prezzi delle materie prime (petrolio in primis), una crisi alimentare mondiale ed una crisi creditizia (seguita a quella bancaria) con conseguente crollo di fiducia dei mercati borsistici.

Secondo Beck (1999), a seguito del processo di globalizzazione che consiste nell'estensione, densità e stabilità delle reti relazionali reciproche regional-globali e della loro auto-definizione massmediale, la dialettica globale/locale non è schematica, anzi, come dice Appadurai (1996) si dovrebbe parlare di un insieme di flussi che attraversa l'intero pianeta, e che riguarda simultaneamente capitale, merci, informazioni, persone, tecnologie ecc. Ecco che anche la crisi può essere letta come un flusso, e a ragione della dicotomia globale/locale si possono leggere fenomeni di significazione all'interno delle strategie economiche sia a livello macro che a livello micro, dove in una logica narrativa gli attanti pongono in essere delle strategie per disgiungersi dall'oggetto crisi; una di queste strategie è un insolito impiego dello spazio.

Secondo la matrice economica occidentale da ogni investimento bisogna trarre un profitto; e l'uso dello spazio commerciale rientra in questa logica;

${ }^{1}$ Si chiama sub-prime il mercato immobiliare formato da soggetti poco agiati che hanno avuto problemi crescenti nel rimborsare le rate dei mutui accesi a suo tempo per comprare la loro casa. 


\section{Ocula ${ }^{16}$}

Semiotics of Economic Discourse | Semiotica del discorso economico

Tiziana Barone| Controtendenza del retail nella crisi del nuovo millennio

ma non è sempre così, poiché vi sono spazi del retail come i temporary store e i suoi affini, che non sempre rispondono alla logica dell'investimento.

In questo contributo andrò ad indagare su alcuni di questi spazi temporanei grazie a delle immagini trovarte on line. Internet con la sua natura immediata ed ipermediata $\mathrm{mi}$ ha permesso di indagare la rappresentazione di uno spazio tramite testi visivi; per tale motivo la rappresentazione non è definita né ontologicamente né oggettivamente, ma formulata in base all'esperienza dello spettatore (Bolter, Grusin, 2006).

\section{Sua maestà la finanza}

Il timore che dalla crisi finanziaria ne conseguisse una dell'economia si è concretizzato, vi è stata infatti contemporaneamente, e soprattutto negli Stati Uniti, una diminuzione del valore della fiducia, fondante per il meccanismo economico della concessione del credito, da cui una restrizione dell'erogazione monetaria alle imprese e alle persone.

Così le aziende, locali, globali o transnazionali hanno implementato delle strategie diverse come le partnership sempre più legate allo scambio di beni e servizi, una maggiore attenzione al sociale e al culturale, il crowdfunding, e nell'ambito dell'uso o del riuso di luoghi un maggiore impiego di spazi a tempo. Questa forma di spazio commerciale esiste già da 15 anni, la modalità d'impiego è cambiata.

Mentre prima era solo una strategia di marketing per giocare sull'effetto sorpresa e usare un approccio soft selling nei riguardi del consumatore, oggi per chi fa retail può essere un'esigenza economica, in particolare in Italia: si risparmia in termini di costo e si massimizza il processo di branding che si offre ai propri consumatori in un arco temporale che va da pochi giorni a un massimo di sei mesi; ma il temporary store non è solo un luogo economico e commerciale, è soprattutto uno spazio vissuto, è un sistema capace di parlare d'altro rispetto a sé. Marrone (2001) si sofferma sulla considerazione che la presupposizione reciproca dei piani: espressione e contenuto di cui parla il linguista danese Louis Hjelmslev, conferma che lo spazio è un linguaggio a tutti gli effetti: esso ci parla della società in cui viviamo e come questa si rappresenti; una società che diventa serbatoio di significati, valorizzazioni, programmi d'azione e di passioni.

\section{Controtendenze: dall'on-line all'off-line}

Il progredire della crisi economica negli ultimi anni ha fatto sì che nell'ambito del retail si innestasse una particolare controtendenza: nella discorsività intrattenuta da un brand varie forme espressive come lo store e il sito web, o il packaging, l'uso dei social ecc. hanno trovato una nuova riconfigurazione. Partendo dalla narratività come principio esplicativo, la crisi può essere letta come un antisoggetto che entra in relazione polemica col soggetto impresa: questa implementerà delle tattiche che consentiranno di disgiungersi dall'attante oggetto, ovvero la chiusura dell'azienda. Alcune di 


\section{Ocula ${ }^{16}$}

Semiotics of Economic Discourse | Semiotica del discorso economico

Tiziana Barone| Controtendenza del retail nella crisi del nuovo millennio

queste tattiche prevedono la chiusura di punti vendita e in generale l'abbattimento dei costi sfruttando al massimo il web e le campagne social. Cambia il contesto economico e le esperienze soggettive che si confrontano con le pratiche economico-sociali modificano lo status della dialettica fra ricezione e trasmissione in ciò che viene chiamato tendenza. La tendenza individua un microuniverso emergente "capace di far convergere verso di sé atteggiamenti e comportamenti" (Ceriani, 2007) che si estendono trasversalmente; una continua tensione tra valori preesistenti e usi della società, i quali perseguono lo stesso ritmo di trasformazione. Ecco che si installa una concezione opposta alla tendenza a ridurre i costi, ovvero una controtendenza ${ }^{2}$; dal punto di vista economico si fanno degli investimenti in retail da parte di aziende che nascono come brand on-line.

Da questa controtendenza che ho riscontrato in questo ambito diverse azioni sono accomunate dalla presenza di un'unica tematizzazione, realizzata dal valore della controtendenza in una doppia accezione: si vuole veicolare la valorizzazione del brand on-line sotto una nuova sostanza 'espressiva ma anche quello di destinazione della distribuzione, nel caso in cui le aziende operino in mercati non locali. È interessante notare come ogni compagnia declini questa valorizzazione in modo diverso, e lo fa sia in base ai propri scopi distributivi che alla propria brand-identity, cioè le aziende cercano di valorizzare la marca stessa e non singoli prodotti.

\section{Una spazialità differente}

Un pop up shop o temporary store è un negozio a tempo realizzato dalle aziende per incrementare la brand awarness, lanciare nuovi prodotti, testare il mercato e creare nuove esperienze multisensoriali.

Il termine pop up è un neologismo che riguarda ogni cosa che sia temporanea; la maggior parte dei brand hanno capito la potenzialità di questi strumenti del retail e ne fanno uso nelle maniere più disparate. I pop up store aumentano la loro popolarità durante grandi eventi o giorni festivi in luoghi molto trafficati o durante eventi di portata globale (Fashion Week nelle varie città, Black Friday ecc.). Altri preferiscono scegliere location più impopolari, ma non per questo meno significanti come Prada che ha aperto un temporary store nel deserto texano (2005), oppure H\&M che installa un pop up container a Scheveningen, località costiera di L'Aia (2011).

Assotemporary, l'associazione italiana dei temporary shop, showroom e business center, definisce il pop up store come "ultimo confine del retail e nuova frontiera della comunicazione" poiché si incardina nella logica "intrattenimento, esperienza, sorpresa".

Il rapporto soggetto-spazio assume un valore diverso rispetto a rapporto che si può instaurare con un flagship o un centro commerciale. Il fruitore del temporary shop, secondo l'assiologia flochiana, nella maggior parte dei casi si colloca sulla deissi utopico/ludico, di conseguenza è spesso un conviviale e

${ }^{2}$ una frattura interna dell'isotopia che si evidenzia tramite la punta dell'iceberg riconoscibile socialmente da un evento. 


\section{Ocula ${ }^{16}$}

Semiotics of Economic Discourse | Semiotica del discorso economico

Tiziana Barone| Controtendenza del retail nella crisi del nuovo millennio

un edonista. Interior, exhibit design e merchandising sono elementi identificativi fondanti dell'architettura e del layout del temporary store a prescindere dalla categoria merceologica proposta.

Il temporary store è ovviamente un testo e come tale può essere analizzato a livelli sempre più profondi. Sul piano dell'espressione gli oggetti sono iscritti nel dispositivo dell'allestimento, a sua volta iscritto nello spazio architettonico. Esistono tre relazioni di presupposizione tra questi termini:

- lo spazio architettonico presuppone gli oggetti e l'allestimento;

- l'allestimento presuppone le merci;

Questi incastri si possono leggere sia da un punto di vista sintagmatico che paradigmatico; secondo il primo approccio prevarrà la logica della contiguità e della concatenazione espositiva, nel secondo approccio prevarrà il sistema di distinzione e gerarchizzazione legato ai prodotti (Floch, 2006).

La paradigmatizzazione dello spazio è rafforzata dall'impiego di luci soffuse: faretti alogeni a luce calda e accostamenti di materiali: dai tradizionali a quelli insoliti, a materie di riciclo. Anche gli effetti cromatici sono di notevole importanza in quanto creano, in determinati casi relazioni di significazione non indifferenti.

L'approccio sintagmatico del piano dell'espressione, ovvero la concatenazione delle qualità spaziali e visive, prevede un ritmo diverso della disposizione architettonica a volte creato appositamente laddove la struttura adibita a store non lo avesse. I cambi di ritmo e tensività spaziale insieme a tutti gli altri elementi espressivi producono un processo passionale al centro del quale si trova il consumatore.

Sul piano del contenuto invece il significato dello spazio è legato alla creazione di relazione sempre più forte con il consumatore che possiamo definire sofisticata, oltre agli eventi, ci si approccia al marketing non convenzionale, all'uso di tecnologie e tecniche di realtà aumentata.

\section{Il temporary store}

Un brand declina la sua comunicazione in varie forme, le cui invarianti semiotiche sinergicamente confluiscono in ciò che i marketer chiamano brand image. In questo contributo esaminerò due internet company che vanno controtendenza rispetto al trend economico degli ultimi anni, ovvero Google e Ebay. Il primo brand propone il Winter Wonderlab (Usa, 2013), mentre il secondo propone 2 spazi differenti: Social Virtual store (Londra, 2012) e Ebay Toy Box (New York, 2013), i quali affiancano ad una struttura di business on-line una struttura spaziale "in cui l'essere umano di muove e svolge la sua attività, per cui è inteso [...] come luogo in cui si dispiega una significazione" (Zunzunegui, 2011, pag. 74).

Un temporary store è un luogo privilegiato all'interno del quale si concretizzano strategie e politiche di comunicazione e marketing, in modo da ridisegnare uno spazio per conquistare il consumatore ponendolo al centro di esso. 


\section{Ocula ${ }^{16}$}

Semiotics of Economic Discourse | Semiotica del discorso economico

Tiziana Barone| Controtendenza del retail nella crisi del nuovo millennio

Cercare di far muovere le strategie comunicative in questa direzione fa sì che il temporary store si proponga come un teatro all'interno del quale tutti gli elementi, dagli arredi al personale di vendita, concorrano a mettere in scena una rappresentazione che consenta al cliente di vivere un'esperienza d'acquisto e di consumo di un determinato bene o servizio (Pastore, Vernuccio, 2012).

Dal punto di vista narrativo lo spazio del temporary store è il soggetto attanziale, il quale per poter portare a termine il suo programma d'azione, che può essere la radicalizzazione della brand affinity mette in atto un programma d'uso: fare branded entertainment 3 .

Nel caso di un flagship o di un temporary (o pop up) store l'organizzazione interna dei percorsi è meno complessa, in alcuni casi l'ambientazione è addirittura unica, e sarà l'omogeneità di alcune caratteristiche delle sostanze espressive interne allo store, come il layout, o installazioni particolari a far capo all'asse del "sistema" (paradigmatico) dell'identità visiva del brand, all'interno del quale si innestano gli assi di "processo" (sintagmatico). Ecco che l'espressione dell'identità visiva dello spazio commerciale viene fuori dalla combinazione di elementi invarianti ed elementi variabili che rientrano nel sincretismo discorsivo e interdiscorsivo.

Le attrezzature di vendita spesso sono modalizzate da un voler non essere viste per dare risalto ai prodotti che vi sono esposti. In altri casi come nel pop up store Google in Bryant Park, il layout espositivo vuole essere visto poiché, oltre ad avere uno scopo funzionale partecipa al gioco ottico di tutto il testo creando, anche attraverso il discorso dell'interior design, un legame isotopico con l'ambiente circostante. Nel caso di Ebay in Covent Garden, layout e percorsi sono funzionali al programma narrativo dell'attante ibrido; mentre nel caso dell'Ebay Toy Box non esiste una soglia da varcare per entrare dentro il negozio, gli acquisti vengono fatti all'esterno. Lo spazio utopico in cui la performance ha luogo è lo spazio che c'è sulla pedana, su cui è poggiato il container, e tra questo e un albero di Natale stilizzato, i cui ornamenti portano iscritti i QR code (fig. 1-2).

\section{Definizioni e confusioni}

Coerentemente ai concetti di fluidità, evanescenza e dinamismo con cui si manifesta la post-modernità, anche l'architettura, disciplina storicamente fondata su un principio di durata illimitata del manufatto architettonico, si è adeguata ad una dimensione temporanea limitata, se non addirittura a "scadenza".

Nel campo commerciale il tradizionale approccio relativo ad una costruzione architettonica strutturata e duratura ha spostato l'attenzione

3 Rientra nel più vasto dominio del content marketing, a volte lo si fa coincidere con il branded content. L'ANA (Association of National Advertisers) definisce: "Branded entertainment (also called branded content) is the convergence of the advertising and entertaiment industries where a brand message is integrated within an appropriate context as a part of the interaction. It is much more than simple product placement, where a product may be visually included, but not discussed." 


\section{Ocula ${ }^{16}$}

Semiotics of Economic Discourse | Semiotica del discorso economico

Tiziana Barone| Controtendenza del retail nella crisi del nuovo millennio

verso le discipline tecnico progettuali che potessero arricchire l'esperienza spaziale con differenti suggestioni. Lo spazio dello store è così diventato un terreno di integrazione disciplinare, che concorre alla costruzione di un'esperienza, non più legata alla sola percezione fisica dello spazio e dei prodotti in esso contenuti, quanto ad una molteplicità di stimoli, sensoriali, narrativi e sociali riassumibili nella cosiddetta brand entertainment.

I termini temporary store e pop up store si usano spesso in maniera interscambiabile, ma sono veramente dei sinonimi? Già dalla traduzione inglese troviamo due sostantivi differenti che conducono a una significazione diversa: il vocabolario online wordreference.com (2015) restituisce le seguenti traduzioni:

- temporary: (not permanent) temporaneo, provvisorio;

- pop up: (figurative, informale) emergere, succedere, saltare fuori

Mentre nel primo caso è il fattore tempo a scandire il significato della parola, nel secondo caso, a significare è la capacità di un soggetto ad apparire improvvisamente; possiamo quindi parlare di sinonimia? Certamente no!

Il termine generale pop up retail è usato, in ambito economico con un'accezione più ampia, e viene utilizzato per descrivere le iniziative che manifestano la tendenza a spuntare senza preavviso, in modo inaspettato, e che altrettanto velocemente spariscono o mutano in qualcos'altro; indica in generale la rottura della continuità temporale prima con la presenza di un allestimento spaziale che fino al giorno prima non c'era, e che poi improvvisamente sparisce. Tale fenomeno sposta il focus della dimensione fisica dello store verso quella immateriale dell'evento e della pura esperienza sociale. Si può quindi affermare che attraverso il pop up retail si ha una prevalenza dell'evento sul luogo.

I temporary store, identificano, invece, i punti di vendita con apertura a tempo determinato. La parola "temporaneo" è diventata di moda, quasi divertente, come a segnalare la bellezza del cambiamento e il carattere di urgenza dell'acquisto odierno imposto dall'obsolescenza estetica.

In questo caso, a differenza del precedente, la data di chiusura è fissata negli obiettivi programmatici dell'azienda o dell'impresa che sostiene il progetto, e viene categoricamente rispettata, a prescindere dagli esiti positivi o negativi in termini economici. Inoltre, tale scadenza viene comunicata apertamente al consumatore già a partire dalla data di apertura, garantendo, pertanto, il venire meno del carattere dell'imprevedibilità.

Ad ogni modo, entrambi sono luoghi praticati in cui si potrà leggere l'esperienza enunciata a cui si sovrappone l'esperienza empirica del consumatore; non solo il fattore tempo scandisce l'esistenza stessa di questo spazio, ma definisce anche il tipo di esperienza che vuole coinvolgere il consumatore, e in ultimo l'azienda, il cui enunciatore, che con la sua messa in discorso traduce il processo narrativo del racconto economico in una forma di controtendenza per sconfiggere la crisi, anche se finanziariamente non sempre è garantito il successo.

Dal punto di vista economico questa sostanza dell'espressione commerciale non solo consente di innovare costantemente il sistema distributivo, grazie al meccanismo passionale basato sullo stupore della 


\section{Ocula ${ }^{16}$}

Semiotics of Economic Discourse | Semiotica del discorso economico

Tiziana Barone| Controtendenza del retail nella crisi del nuovo millennio

sorpresa, mettendo in risalto tensività e aspettualità permettendo grazie alla tempistica molto limitata, di dotarlo di una forma di esclusività agli occhi dei consumatori.

Prima di passare al Winter Wonderlab e agli Ebay pop ups' ritengo opportuno soffermarmi sull'analisi della relazione spazio/tempo; già Lotman (1980) ci parla della facoltà di un'interruzione provvisoria e trasversale che rende conto dei flussi diversi e asincronici che lo compongono chiedendo una chiave di lettura di volta in volta adattata (Ceriani 2013). È questo il punto di partenza, se guardiamo il piano logico narrativo avremo l'opposizione continuità $v s$ discontinuità del tempo:

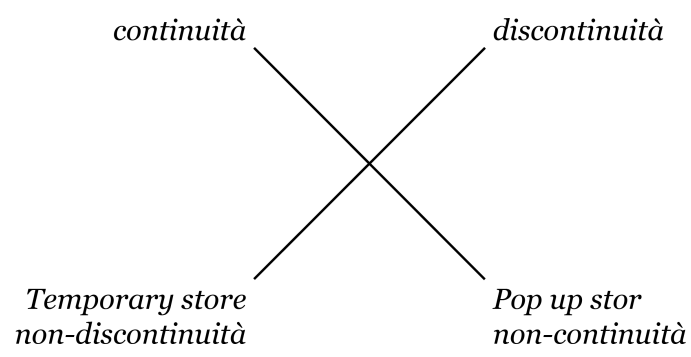

I poli di opposizione affermativa non consentono in questo caso di lavorare sulla significazione del pop up retail, spostandoci sull'asse dei sub contrari invece si vede come lo spazio commerciale si descriva come forma di narrazione dove ogni trasformazione sviluppa inter e intratestualmente la relazione tra narratività e discorsività, e viceversa (Ceriani, 2013). La vita di quello spazio che abbiamo definito pop up store si colloca doppiamente nella non-continuità del tempo. La doppia accezione di non-continuità è dovuta sia al ciclo di vita (in senso economico) dello spazio pop up store in relazione al suo co-testo4, ma anche alla sovrapposizione, sul piano del contenuto, dell'esperienza sociale e della brand entertainment, le cui manifestazioni espressive sono dovute rispettivamente agli eventi organizzati e all'articolazione spaziale dello store; chi valorizza la non-discontinuità attende l'inatteso e ama tutto quanto può sorprenderlo, colpirlo o rallegrarlo (Floch, 1990). Sull'asse della non-discontinuità invece si colloca il temporary store il quale valorizza la sospensione o l'interruzione, ma non la rottura; la strategia della non-continuità è quella dell'incastro, della trasgressione. Ecco che la data di scadenza fissa un limite nell'esperienza proposta dalle aziende; questo limite non è solo temporale ma è anche socio-economico e porta con sé le tracce enunciative di pratiche diverse, fermo restando che trattiamo lo studio delle pratiche come dei testi.

4 Ogni elemento spaziale assume significato in base al tipo di relazione (sintagmatica o paradigmatica) che intrattiene con gli altri elementi che compongono lo stesso sistema. Secondo Lotman (1998): "[...] qualsiasi testo complesso può essere considerato come un sistema di sottotesti, per i quali esso rappresenta il contesto, uno spazio entro cui si compie un processo di formazione semiotica di significato" (Giannitrapani, 2013) 


\section{Ocula ${ }^{16}$}

Semiotics of Economic Discourse | Semiotica del discorso economico

Tiziana Barone| Controtendenza del retail nella crisi del nuovo millennio

\section{Google}

Uno dei brand più conosciuti al mondo che nasce on-line ed ha una forte identità costruita essenzialmente sulla semplicità del motore di ricerca, a cui, nel corso del tempo sono state aggiunte molteplici funzioni: i social, il Maps, Google Earth, il Google Art-project e la vendita di device brandizzati è Google. La società quotata in borsa da circa 10 anni, propone una nuova forma di contatto con i suoi utenti attraverso spazi a tempo. Negli Stati Uniti nel novembre 2013, quando il paese stava già uscendo dalla crisi grazie alle politiche di Obama, Google lancia una nuova modalità di esperienza di consumo in un pop up store a tema dove in realtà non si compra nulla durante la visita: è il Winter Wonderlab. Già dal nome si rileva una forte presenza isotopica con il mondo delle fiabe, in particolare con Alice in the Wonderland, ma sta volta land è sostituito da lab, proprio ad enunciare la forma d'esperienza che si andrà a fare.

Il vocabolario Treccani definisce il termine laboratorio come: "Locale o edificio fornito di apposite installazioni e apparecchi per esperienze $e$ preparazioni fisiche, chimiche, farmaceutiche o, in genere, per studi, ricerche ed esperimenti tecnici o scientifici [...].

Partendo da questa definizione è evidente come l'abbreviazione inglese lab modalizza un poter-fare in termini di interazione tra soggetti umani e non umani per realizzare la l'affinity.

Google apre in 6 città diverse degli Stati Uniti all'interno dei centri commerciali della catena Westlife: Maryland (Westfield Annapolis), Chicago (Westfield Fox Valley), California (Westfield Topanga e Westfield Galleria), e New Jersey (Westfield Garden State Plaza), infine New York presso Bryant Park. Qui Google ha costruito una struttura in vetro, molto simile ad una serra.

Il colosso americano si dà la possibilità di contatto con il pubblico implementando due strategie enunciative differenti: nel caso di Bryant Park il testo pop up store è fortemente condizionato dal contesto anche se presenta dei confini ben precisi: lo store che ostenta la sua presenza e si autospettacolarizza grazie al vetro si vuol far vedere, è un informatore del mondo Google nei confronti dell'osservatore Bryant Park e dei suoi utenti, mantenendo una coerenza comunicativa dello spazio stesso e integrandosi con il discorso architettonico circostante. Tale regime discorsivo è in relazione con altri discorsi della città di New York, ma anche con altri store che presentano le medesime caratteristiche in termini di materiali come il flagship di Apple sulla 5th Avenue, in linea d'aria alle spalle di Bryant Park (fig. 3). La stessa relazione viene intrattenuta dal testo con il Googleplex a Silicon Valley.

I pop up store aperti dentro Westlife (fig. 4-5) invece sono spazi eterotipici come del resto tutto il centro commerciale, per cui le pratiche dei fruitori sono già consolidate, e l'elemento sorpresa non è più inaspettato. $\mathrm{Si}$ sa che all'interno di un mall si troverà un artefatto che permetterà ai fruitori di esperire momenti ludici, estendere l'iniziativa commerciale in luoghi di 


\section{Ocula ${ }^{16}$}

Semiotics of Economic Discourse | Semiotica del discorso economico

Tiziana Barone| Controtendenza del retail nella crisi del nuovo millennio

questo tipo permette a Google di massimizzare un gran numero di utenti in poco tempo e con minori costi. Il sintagma di tutti i pop up store all'interno di un mall Westlife è abbastanza omogenea (fig. 6) rispettando a pieno quelle che sono le caratteristiche di un brand globale5.

\subsection{Bryant Park}

Il Winter Wonderlab sorge vicino gli stand natalizi, a ridosso della pista di pattinaggio a Bryant Park (fig. 7-8-9); situato sul lato nord-ovest del parco, lo spazio minimalista il cui arredo composto da espositori e display in plastica, alcuni dei quali presentano una forma irregolare (a simulare blocchi di ghiaccio) (fig. 10), espone gli ultimi prodotti suddivisi in aree tematiche: musica, giochi, browsing ecc. Questi sono a disposizione del pubblico per essere usati, ma la punta di diamante dello store è lo Snow Globe a grandezza naturale posto al centro dello spazio (fig. 11). Varcando la soglia del pop up store, ovvero un dispositivo di passaggio che modifica il ruolo tematico di chi l'attraversa (Giannitrapani, 2013), il flâneur si trasforma in potenziale consumatore, e l'igloo, a sua volta trasforma questo soggetto nel protagonista di una esibizione, una star la cui performance può essere condivisa on-line: filmarsi nel paese invernale delle meraviglie Google con lo slow motion! (fig. 12)

Lo spazio di Bryant Park può, ovviamente essere letto come un sistema di relazioni sintagmatiche, per cui il pop up store, in quanto elemento di questa relazione assume significato in base a ciò che lo circonda; ed ecco che la tensostruttura trasparente mostra il suo interno e al contempo apre visuali esterne sul contesto urbano.

Prima di passare all'analisi interna dello store, o meglio dell'esperienza che viene enunciata in questo spazio, è opportuno fare una riflessione sulle relazioni che intercorrono tra la struttura del pop up store e gli utenti del parco, in particolare ai materiali trasparenti con cui è fatta la struttura mobile e i giochi ottici che si vengono a creare. Floch spiega che il materiale può essere considerato come un oggetto di senso, come la manifestazione particolarmente suggestiva di una riflessione sul mondo del sensibile, di una "logica concreta" in atto; Hammad (2002) si riferisce al vetro come a un materiale che, se posto come "frontiera", ovvero come elemento di separazione fra due spazi, agisce sul regime del permesso e dellinterdetto (Mangiapane, 2008). In questo caso i murib dello store, essendo in vetro agiscono selettivamente: lasciano passare la luce ma riparano dagli agenti atmosferici, permettono una congiunzione sul piano visivo con ciò che accade dentro ma ne inibisce una congiunzione fisica a meno di varcare la

\footnotetext{
5 Un brand è globale quando operano sul mercato mondiale per eccellenza, cioè internet (o hanno una rete distributiva sparsa per i cinque continenti). Questi brand spesso decidono di utilizzare elementi figurali più facilmente declinabili nelle forme culturali con cui entrano in contatto in modo da avere un effetto moltiplicativo della significazione.

${ }^{6}$ Il muro costituisce un limite che interdice qualsiasi comunicazione fra i due spazi
} 


\section{Ocula ${ }^{16}$}

Semiotics of Economic Discourse | Semiotica del discorso economico

Tiziana Barone| Controtendenza del retail nella crisi del nuovo millennio

soglia e trasformare il proprio stato. Questo meccanismo di interdizione selettiva, ad ogni modo innesca delle azioni, ovvero promette o minaccia, persuade o dissuade, in funzione del fatto che l'oggetto o la situazione visti vengano valorizzate positivamente o negativamente (Mangiapane, 2008). Anche le passioni si intrecciano alle azioni, e secondo un regime di visibilità in cui sono coinvolti i due soggetti, in questo caso gli utenti di Bryan Park e il Winter Wonderlab. Secondo la modalità del volere il pop up store vuole essere visto, ma non è detto che gli utenti vogliano vedere. Partendo dal lavoro di Floch sull'analisi dei tragitti degli utenti della metro parigina grazie all'articolazione della categoria semantica discontinuità vs continuità si può ipotizzare un "voler vedere" come modalizzazione di partenza dell'esploratore di fronte ad un "voler non vedere" del sonnambulo, che attraverserà la strada per abitudine, impermeabile a qualsiasi suggestione. Ancora, il bighellone potrebbe attraversare la strada, disponibile a farsi catturare dal muro in vetro posizionandosi su un "non voler non vedere", mentre il professionista sarà interessato solo alle vetrine che lo interessano $\mathrm{e}$ sarà disinteressato a tutto il resto su un "non voler vedere" (Mangiapane, 2008):
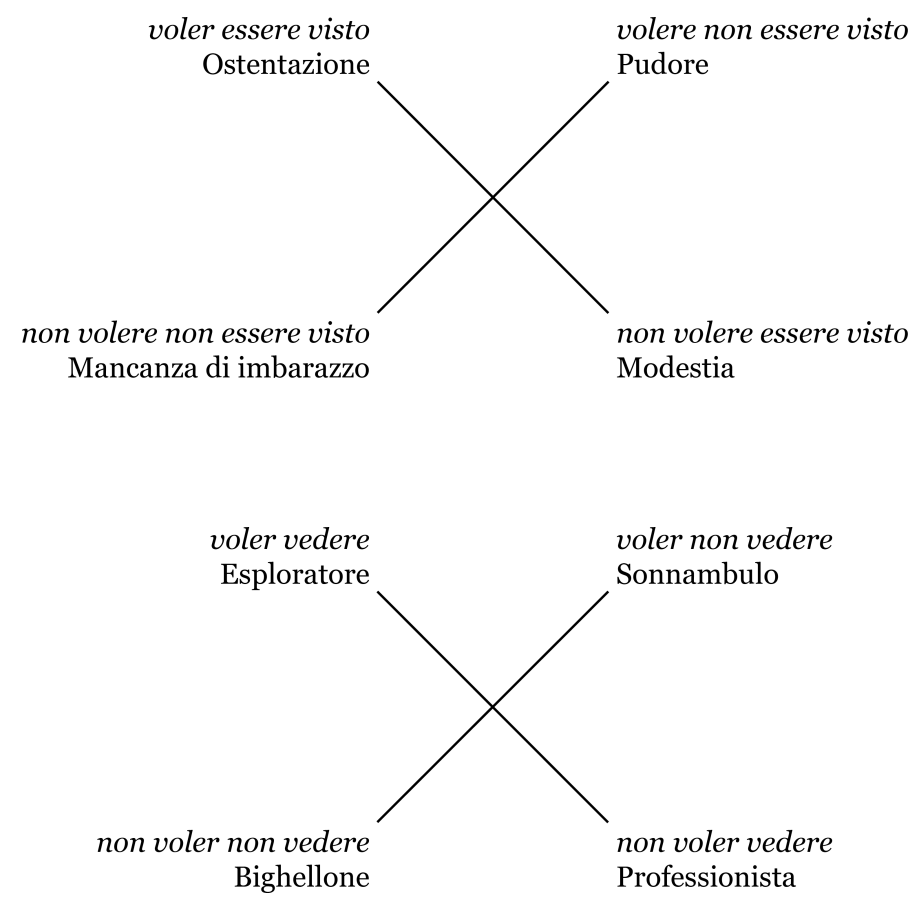

Per cui lo store sicuramente ostenta la sua presenza e attrae gli esploratori, ma anche i bighelloni. È importante notare che il Wonderlab non solo ostenta la sua presenza, ma grazie alla trasparenza ostenta ciò che accade dentro e in particolare la presenza dello Snow Globe, perno centrale del brand entertainment. Per potere fare questa esperienza il visitatore ha bisogno di un aiutante: lo "snowpass" - una carta verde acqua disponibile su 


\section{Ocula ${ }^{16}$}

Semiotics of Economic Discourse | Semiotica del discorso economico

Tiziana Barone| Controtendenza del retail nella crisi del nuovo millennio

tutti i tavoli espositivi e toccare con questo sul retro di un qualsiasi Nexus 7 per iscriversi (fig. 13-14-15). Basta inserire il proprio nome, indirizzo email e numero di telefono cellulare, non appena sarà giunto il proprio turno per entrare nel Globe, saranno inviati due avvisi: e-mail e messaggi di testo.

Infine bisogna dotarsi dei travestimenti a tema natalizio, riposti in apposite scatole (ad esempio un paio di corna di renna) ed entrare nell'igloo e attendere la nevicata con fiocchi di tessuto, aspettare qualche secondo e la telecamera riprenderà in slow motion producendo un effetto Matrix nella ripresa a cui è possibile aggiungere la scelta della musica, quindi condividere il video tramite i social network (Google+, Facebook, Twitter) o inviarlo per e-mail; è difficile non sorridere quando ci si guarda mentre si lanciano vertiginosamente palle di neve in aria a 10 fotogrammi al secondo.

L'esperienza che ho sommariamente descritto in questa sede, in un discorso economico sembra avere qualcosa di anomalo: allinterno dei Wonderlab Google non si vende e non si acquista nulla, al massimo si può ordinare e poi ricevere la merce a casa. Allora perché investire parecchi dollari in realizzazione dello store e nelle attrezzature?

Facciamo un passo indietro.

L'economista Sloman (2007) sostiene che l'economia esamina tutto ciò che ha a che fare con il processo di soddisfazione dei bisogni materiali dell'uomo, e come questi possono essere soddisfatti grazie all'incontro dei desideri umani, che sono illimitati (domanda) e l'insieme combinato di risorse, che invece sono limitate (offerta), bisogna creare equilibrio economico. Da qui si intuisce come l'economia al pari della lingua e della parentela è un vero e proprio sistema nel quale le relazioni tra le varie componenti costituiscono e trasformano le componenti stesse; queste relazioni sistematiche sono di natura narrativa in quanto i soggetti che fanno parte di questo sistema costruiscono, decostruiscono e ricostruiscono la propria identità in funzione di altri soggetti con cui entrano in relazione ora contrattuale ora polemica.

All'interno di questo sistema c'è ovviamente la presupposizione reciproca dei piani espressione e contenuto, da una parte il piano dell'espressione ovvero tutto il processo produttivo che va dall'approvvigionamento delle risorse all'immissione sul mercato di un determinato prodotto o servizio, e il piano del contenuto in cui si ritrovano valori7 ideali e pratici, sia per coloro che producono che per coloro che ricevono.

In economica, come in qualsiasi altro sistema di significazione interagiscono le due diverse forme dell'esperienza umana: la dimensione sensibile (la prassi economico-produttiva) e la parte intellegibile (valori economici e della marca). All'interno della sistematicità economica si ritrovano due grandi aree di interesse riportate in tutti i libri di economia: macroeconomia e microeconomia; al di là dei prefissi nella prima accezione verranno studiati l'insieme della domanda aggregata e dell'offerta aggregata ${ }^{8}$

\footnotetext{
7 In questo caso "valore” ha un'accezione semiotica.

8 Per domanda aggregata si intende la spesa totale realizzata dall'economia, da parte dei consumatori, anche di quelli stranieri che domandano le nostre esportazioni,
} 


\section{Ocula ${ }^{16}$}

Semiotics of Economic Discourse | Semiotica del discorso economico

Tiziana Barone| Controtendenza del retail nella crisi del nuovo millennio

e il sistema economico in genere. La microeconomia si occupa degli agenti individuali che operano nel sistema economico, per cui i singoli consumatori, le imprese, le banche ecc. Gli economisti sostengono che la macroeconomia studia la determinazione della produzione nazionale e la sua crescita nel tempo determinando "l'equilibrio tra risorse e desideri" (visione classica); mentre la microeconomia studia i comportamenti e le conseguenze dei singoli attori sulla base del paradigma costo-opportunità. Chiaramente questo approccio è molto lontano da quello che la semiotica, il marketing e le discipline sociali hanno dimostrato nel passaggio dall'homo economicus all'homo ludens per il quale la razionalità lascia il posto al paradosso, all'incertezza e alla mutabilità (Fabris, 2003).

L'economia si occupa principalmente delle strutture della produzione e del consumo, ma si occupa anche del denaro ovvero costi sostenuti per i diversi beni, retribuzioni dei lavoratori ecc. In generale si occupa della quantità di moneta totale presente nell'economia. Meno chiara e autoevidente è la definizione di finanza, e ancora meno lo è la sua relazione e distinzione nei confronti dell'economia "reale". È importante a questo punto fare una distinzione tra gli elementi di connessione fra economia reale e finanza cioè moneta e denaro, che spiegherò più avanti, e capire come, nel XXI secolo accanto all'economia il ruolo della finanza è diventato determinante nell'aggravarsi della crisi economica che conosciamo, che non è stata l'unica della storia occidentale, ma ha qualcosa di diverso proprio perché ha una natura strettamente finanziaria.

È necessario comprendere l'essenza del concetto di scambio e degli elementi che popolano i due "mondi", cioè le merci (beni e servizi), da un lato, e i prodotti finanziari, dall'altro. Ma soprattutto si tratta di definire con maggiore precisione che cosa sono la moneta e il denaro, poiché questi rappresentano l'effettivo trait d'union fra economia reale e finanza ${ }^{9}$ (Turri, 2013). Queste relazioni interoggettive e intersoggettive sono estremamente variabili soprattutto con l'evolversi del tempo.

Il denaro diversamente dalla moneta, è atemporale e aspaziale, non ha una data di nascita precisa, inoltre ciò che consideriamo denaro in Italia, è denaro anche in Groenlandia o in Burundi. Per la moneta è invece possibile ricostruire, anche se a volte in modo approssimativo, la nascita; essa ha una validità circoscritta a precisi ambiti territoriali, ed è quindi definita temporalmente e spazialmente ${ }^{10}$.

dello Stato e delle imprese che acquistano produttivo o materie prime. Con offerta aggregata si intende l'intera produzione di beni e servizi da parte dell'economia (Sloman, J. 2007).

9 La riserva di valore rappresenta la possibilità di accantonare un mezzo di scambio con la certezza istituzionalmente garantita che esso conservi inalterato il suo valore nei termini dell'unità di conto; a conferma di ciò c'è la caratteristica di convertibilità della moneta, venendo meno questa caratteristica viene sostituito tutto il sistema di scambio basato sulla sostanza dell'espressione monetaria.

${ }^{10}$ Il progressivo processo di smaterializzazione della moneta (assegni, bonifici, carte di credito, bancomat ecc.) non deve quindi essere confuso con il concetto di denaro. 


\section{Ocula ${ }^{16}$}

Semiotics of Economic Discourse | Semiotica del discorso economico

Tiziana Barone| Controtendenza del retail nella crisi del nuovo millennio

Il denaro è quindi la parte intellegibile di quello che è la moneta ovvero una delle sostanze dell'espressione dello scambio, ad esempio il baratto può fare a meno della moneta, ma non del denaro, in quanto quest'ultimo ha un valore simbolico. Nella nostra cultura di riferimento (XXI secolo in Europa e Stati Uniti) il denaro è stato fondante del sistema finanziario. Il denaro è tutto ciò che, nel tempo e nello spazio, ha assunto sostanze espressive diverse in termini di riserva di valore e di ricchezza, inclusa la moneta. L'essere riserva di valore consente alla moneta di essere riconosciuta in quanto forma del denaro e ne rappresenta il legame, assume la caratteristica di essere denaro solo quando la si aliena: conchiglie, riso o sale, così come la moneta cartacea o quella elettronica assumono la caratteristica di essere denaro solamente privandosene.

Tornando al Winter Wonderlab di Google si assiste ad uno scambio tra il brand e i consumatori, le cui contropartite sono: da una parte l'esperienza tattile dell'uso dei device Google e ovviamente la possibilità di girare il video nello Snow Globe innescando la viralità on-line e lo share, ovvero il meccanismo del passaparola elettronico, quindi una narrazione nella narrazione, e dall'altra, i consumatori "pagano" Google con i propri dati, brand affinity e brand media, ovvero le sostanze espressive dello scambio, mentre sul piano del contenuto si ritrova il prezzo relativo ${ }^{11}$.

\section{Ebay}

Ebay Inc. è una internet company che si propone sul mercato globale con un sito di aste on-line fondato nel 1995; in Italia è arrivato nel 2001 rilevando il sito iBazar.

Ebay è una piattaforma web (marketplace), molto simile ad un sito di ecommerce, che offre ai propri utenti la possibilità di vendere e comprare oggetti sia nuovi sia usati, in qualsiasi momento, da qualunque luogo e con diverse modalità, incluse le vendite a prezzo fisso e a prezzo dinamico, comunemente definite come "aste online". Anche questa azienda, andando controtendenza, da poco più di sei anni propone i suoi pop up store in varie parti del mondo, con un design sempre diverso ed eterogenero. Si possono trovare in Asia, nelle capitali europee e negli Stati Uniti, all'interno di una struttura architettonica già esistente o tramite l'utilizzo di container o mezzi di trasporto.

In questo contributo proporrò due pop up store di Ebay, uno del 2012 aperto a New York e uno aperto a Londra nel 2013.

\subsection{Ebay Social Shopping in Covent Garden - Londra}

Nel dicembre del 2012, a Londra, nella piazza principale di Covent Garden, nei pressi dell'Apple store (aperto 2 anni prima) viene assemblato un container pop up box, formato da 5 elementi singoli, 4 affiancati disposti trasversalmente e uno sopra questi come se fosse un coperchio, con un

${ }^{11}$ Valore del rapporto di scambio tra due beni. 


\section{Ocula ${ }^{16}$}

Semiotics of Economic Discourse | Semiotica del discorso economico

Tiziana Barone| Controtendenza del retail nella crisi del nuovo millennio

grosso fiocco rosso stampato e un "biglietto d'auguri" recante al frase: "from Ebay with love" seguito dall'hashtag \# ebaysocialshopping (fig. 16).

È la piazza più cool di Londra a fare un dono ai suoi astanti, dono che, in sé, contiene un'esuberanza di valore rispetto alla natura dello spazio; Barthes a tale proposito quando descrive il pacchettino giapponese (L'impero dei segni, 1970) esalta il valore attraverso la promessa che fa l'involucro il quale racchiude spesso un oggetto insignificante di cui "rimanda a un dopo" improvviso, la scoperta. Desiderio, capriccio, interesse possono spingere il flâneur ad entrare nel pop up producendo una sorta di autogratificazione; anche eventuali acquisti che ciascuno fa per sé stesso si possono collegare alla logica del dono e immaginare di poter inscrivere molti consumi (almeno in chiave retorica) nell'economia del dono - Regàlati l'esperienza del prodotto X! In tal modo, fare una certa esperienza diventa un indicatore dello stile di vita del consumatore, e soprattutto mezzo di riconoscimento di un'identità profonda. La relazione tra il pop up store di Ebay e gli utenti di Covent Garden contiene sempre più un principio di reciprocità, di dono incrociato: il dono e l'obbligo che esso crea, il surplus di valore offerto in cambio della brand loyalty.

Ovviamente lo store, che ha tutte le caratteristiche descritte precedentemente, di un pop up, valorizza la non discontinuità del tempo, e lo fa con il suo brevissimo ciclo di vita di appena un fine settimana e con una modalità che vuole anticipare il futuro del retail. Questo spazio può essere definito"negozio virtuale dinamico" e unisce alla nuova tendenza in negozi virtuali in cui non vi è fisicamente alcun prodotto, ma solo le loro immagini proiettate sulle pareti, e il QR code con una sorta di piccola istruzione per l'uso o l'acquisto come fa Amazon, basandosi sui rumors dei social. Tecniche di realtà aumentata, e informazioni che viaggiano sui social network, hanno uno scopo ben preciso per Ebay, cioè fare in modo che intorno al brand si consolidi una comunità di utenti che si identifichino con i valori della marca. Questi soggetti, nel momento in cui varcano la soglia dello store subiscono una trasformazione, come accade per il Winter Wonderlab. Il flâneur, in termini narrativi, non è altri che un attante ${ }^{12}$ ibrido, ovvero un uomo con lo smartphone per cui il mondo assume un significato differente in base alla relazione esistente tra umani e non umani. Latour (1992b) ci parla di traduzioni, ovvero il passaggio di competenze e di forme di manipolazione semiotica che hanno luogo quando soggetto e oggetto si incontrano, il venire meno di uno dei due ferma questa catena di traduzioni perdendo di vista il fenomeno che abbiamo davanti (Mangano, 2008). In questo caso l'ibrido avendo la capacità d'agire percepisce il brand entertainment in maniera diversa poiché, tramite una catena continua di débrayage ed embrayage trasforma un fenomeno sociale come il consumo, in una nuova forma di interazione. Infine questa si conclude con la performance, cioè con la

12 Bruno Latour sostiene che l'uomo con l'agenda elettronica è un attante, cioè esseri definiti da un certo programma d'azione più che da una loro presunta natura (Mangano, 2008) 


\section{Ocula ${ }^{16}$}

Semiotics of Economic Discourse | Semiotica del discorso economico

Tiziana Barone| Controtendenza del retail nella crisi del nuovo millennio

scansione del QR code per l'acquisto, la merce arriverà direttamente a casa (fig. 17).

\subsection{Ebay Toy Box in Meatpacking District - New York}

Nel dicembre del 2013 Ebay apre un nuovo pop up store, questo spazio dal ciclo di vita di appena 4 giorni, sorge di fronte l'Apple Store a Meatpacking District NY (fig. 18); una zona totalmente riqualificata dal riuso di fabbriche ed ex mattatoi, diventata uno dei poli culturali grazie a musei (Whitney Museum) e nuove strade dello shopping, nonché la presenza di un prato sopraelevato su un percorso di una vecchia ferrovia, ora solo pedonale (fig. 19). Questo distretto dopo la riqualificazione ha cominciato ad assumere significazione proprio in opposizione alla NY dei grattacieli, a quell'isola-logo della città che è Manhattan; alla vita frenetica che vi si svolge si oppone "la misura d'uomo", il piacere di passeggiare e godersi lo spazio circostante, la libertà di assaporare lentamente il proprio tempo anche durante la pausa caffè. Il concept pop up Toy Box intrattiene un discorso col Meatpacking che lo contiene, dal momento che lo scopo di Ebay è vendere giocattoli che vanno in beneficenza per la fondazione Toy for ToTs che si occupa di bambini disagiati; installare una grande scatola regalo con un fiocco verde sopra (fig. 20-21), e una parete vetrina con tutti i giochi che si possono acquistare sempre con la modalità del $\mathrm{QR}$ code descritta in precedenza, dà la possibilità ai newyorkesi, non solo prendersi il tempo per sé stessi, ma addirittura per gli altri: compiere una buona azione sotto Natale. Lo store è stato inaugurato dall'attrice statunitense Jenny Garth come testimonial dell'iniziativa (fig. 22), 3 anni prima invece era stata Debra Messing, anche il cantante e attore Darren Criss ha dato il suo contributo con una serata di beneficenza davanti allo store (fig. 23).

L'azienda Ebay anche in questo caso crea comunicazione con il passaparola elettronico, usando i social come cassa di risonanza di un approccio al retail, e ottiene tre vantaggi economici; al denaro sul piano del contenuto corrisponde un sincretismo espressivo dovuto a:

1. ricavi monetari della vendita di prodotti o servizi;

2. informazioni sui clienti

3. aumento di brand awarness, brand loyalty e brand affinity relative ad un miglior posizionamento grazie ad azioni di beneficenza legate alla corporate social responsability.

\section{Conclusioni}

Da questa breve panoramica si evince come il discorso economico e il discorso dello spazio siano interrelati, anche se le aziende usano strategie enunciative differenti, le strutture narrative sono le stesse: sia Google cheEbay si disgiungono dall'oggetto crisi.

Dal punto di vista del discorso economico entrambe aumentano la propria riserva di valore, e grazie alla controtendenza si avrà che il denaro (la 


\section{Ocula ${ }^{16}$}

Semiotics of Economic Discourse | Semiotica del discorso economico

Tiziana Barone| Controtendenza del retail nella crisi del nuovo millennio

parte intellegibile dello scambio) sarà dato dal brand entertainment, mentre la parte sensibile sarà data in larga parte dalle informazioni, ma anche dai ricavi monetari.

Le nuove forme di retail, insieme a tanti altri fattori, contribuiscono quindi a variare le strutture profonde del sistema economico basato sul regime relazionale tra marca e scambio.

\section{Immagini}



Figura 1. Albero dell'Ebay Toy box



Figura 3. Apple Store in $5^{\text {th }}$ Avenue

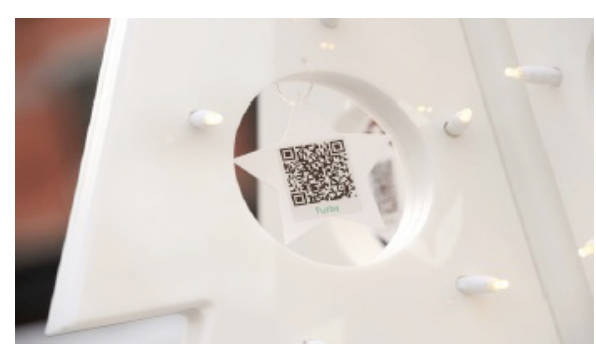

Figura 2. Particolare del QR Code

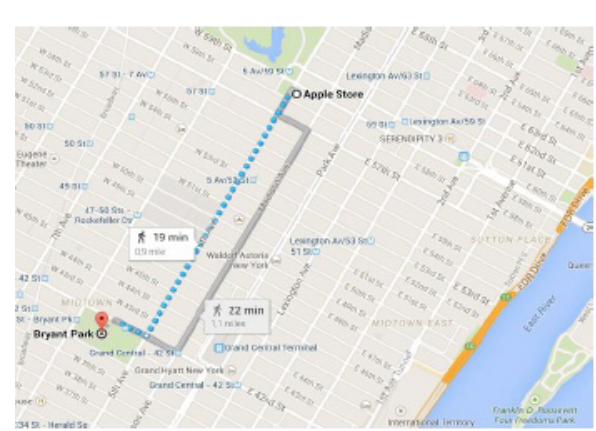

Distanza tra Winter Wonderlab in Bryant Park ed Apple Store nella $5^{\text {th }}$ Avenue 


\section{Ocula ${ }^{16}$}

Semiotics of Economic Discourse | Semiotica del discorso economico

Tiziana Barone| Controtendenza del retail nella crisi del nuovo millennio
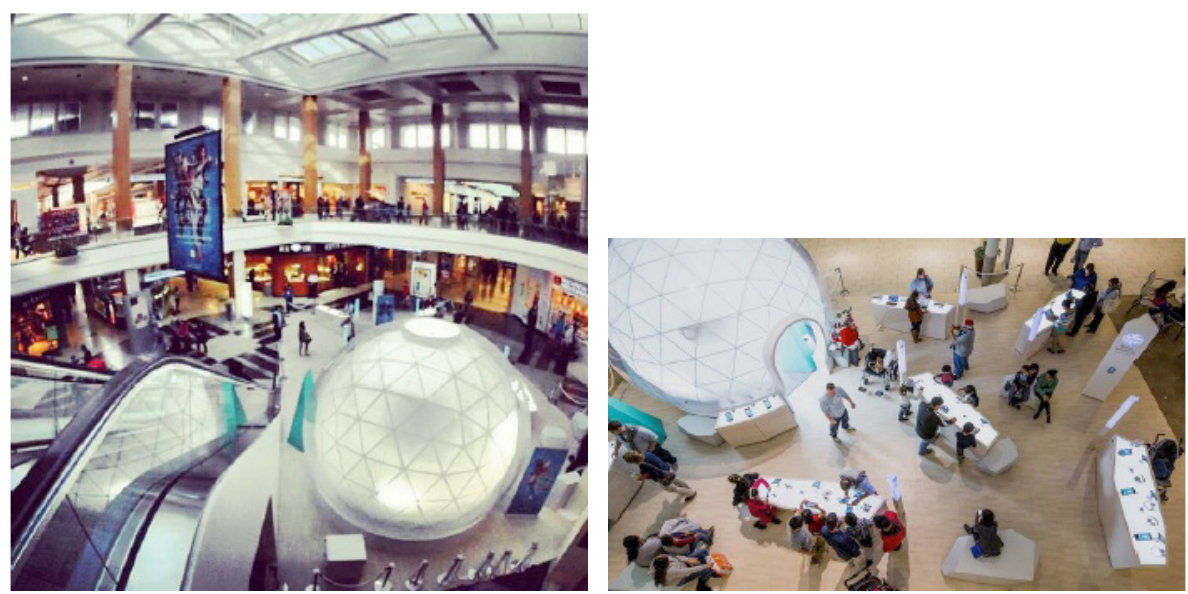

Figura 4

Figura 5
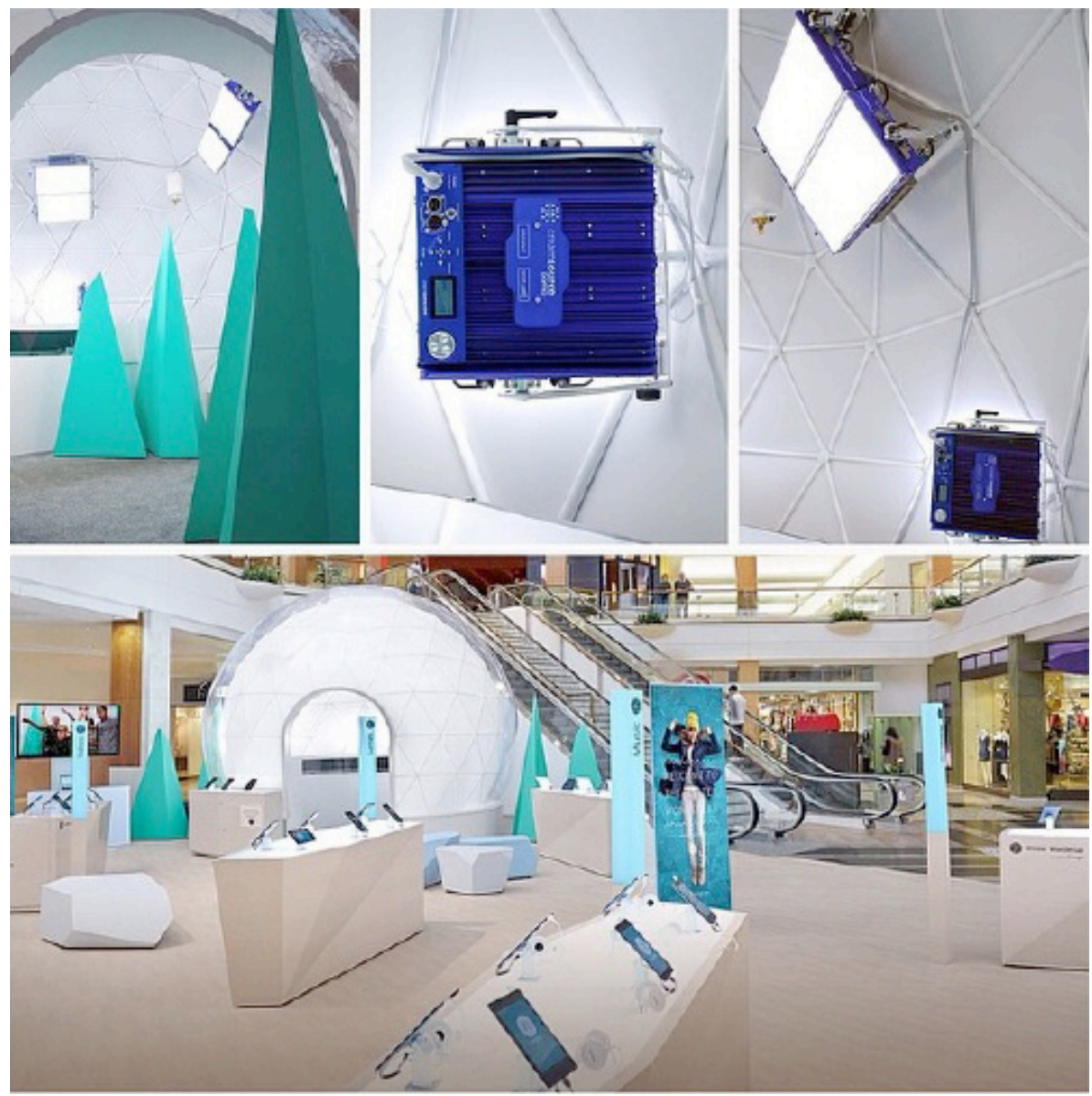

Figura 6. Particolari dei pop up store Google all'interno dei mall Westlife 


\section{Ocula ${ }^{16}$}

Semiotics of Economic Discourse | Semiotica del discorso economico

Tiziana Barone| Controtendenza del retail nella crisi del nuovo millennio

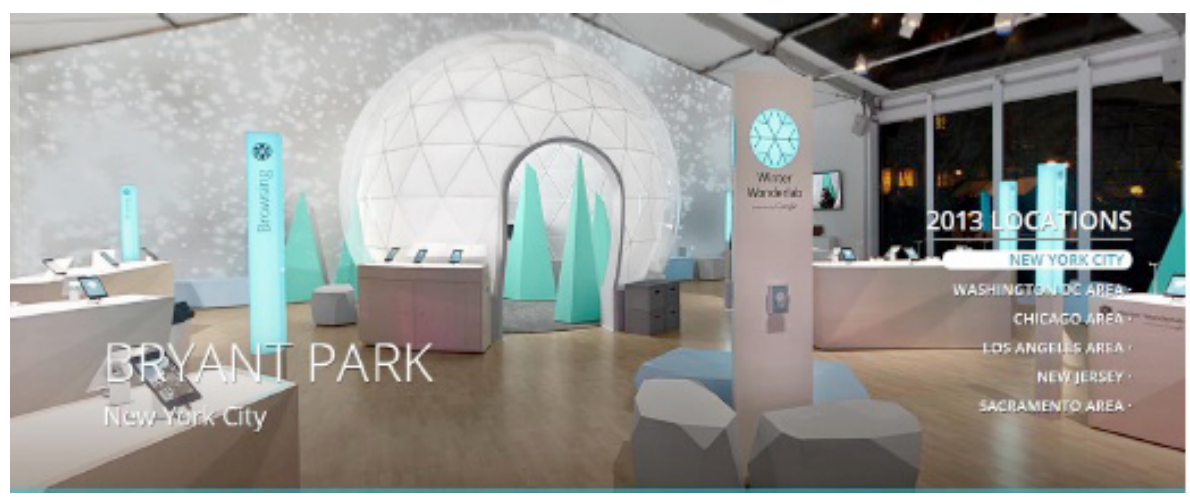

Figura 7 . Foto tratta dal sito web ufficiale del Google Winter Wonderlab

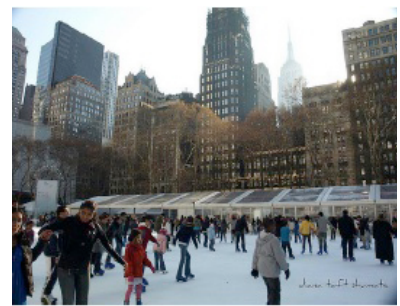

Figura 7.1 Pista di pattinaggio Bryant Park $N Y$

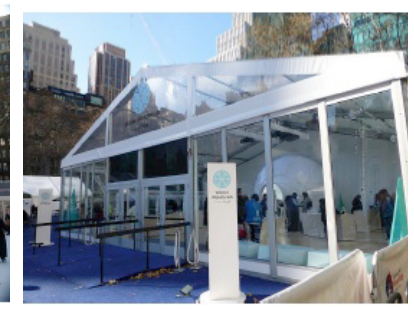

Figura 8. Winter Wonderlab in Bryant Park farsi vedere



Figura 9. Lo store vuole

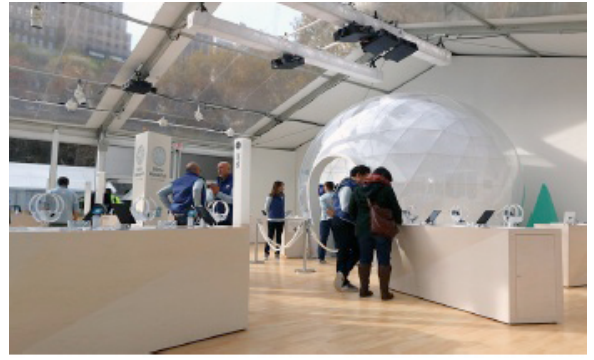

Figura 10.

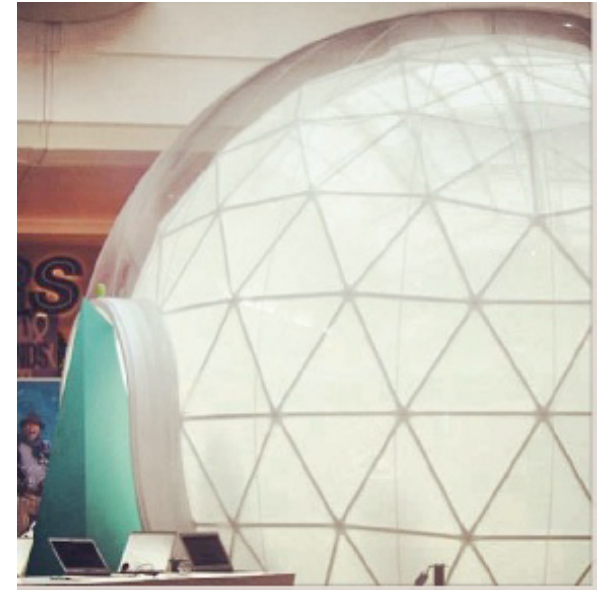

Figura 11. 


\section{Ocula ${ }^{16}$}

Semiotics of Economic Discourse | Semiotica del discorso economico

Tiziana Barone| Controtendenza del retail nella crisi del nuovo millennio

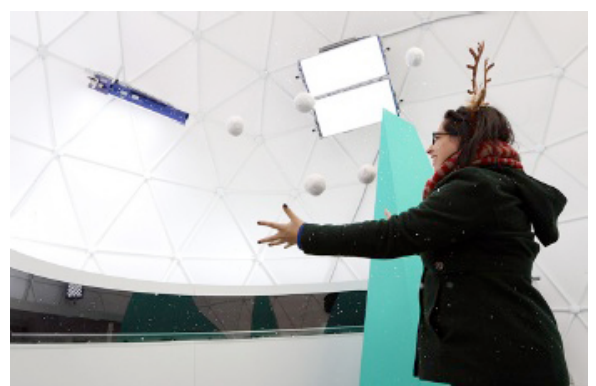

Figura 12.

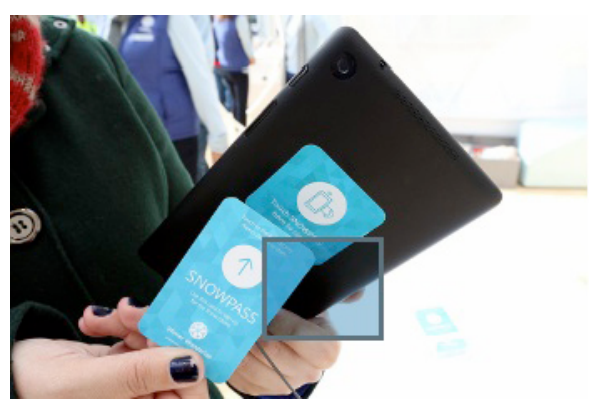

Figura 14.

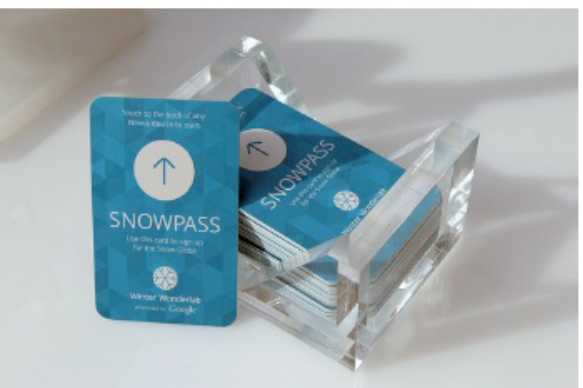

Figura 13.



Figura 15 .

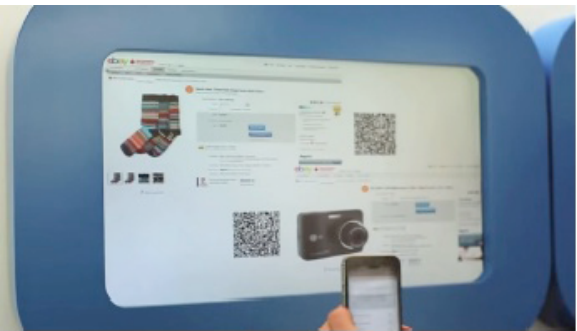

Figura 17.

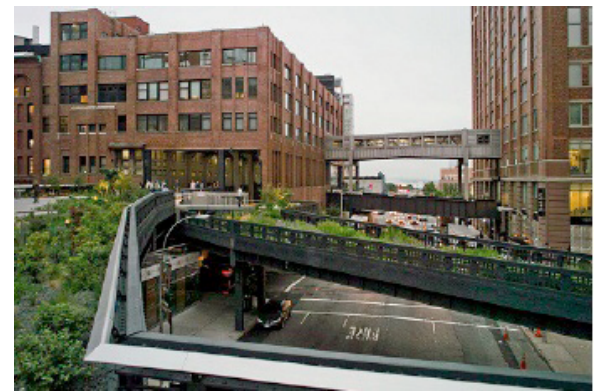

Figura 19.
Figura 18.

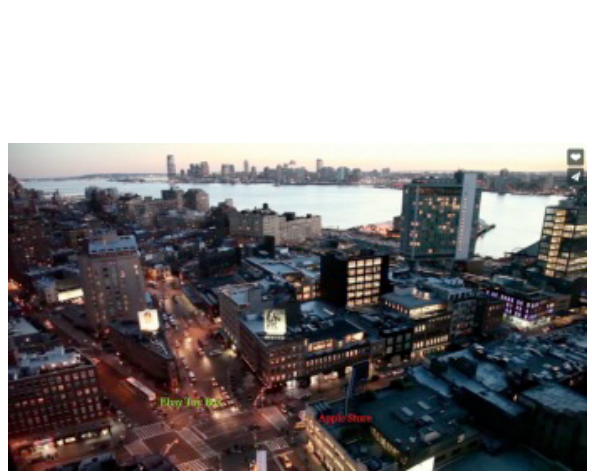

Figura 16.

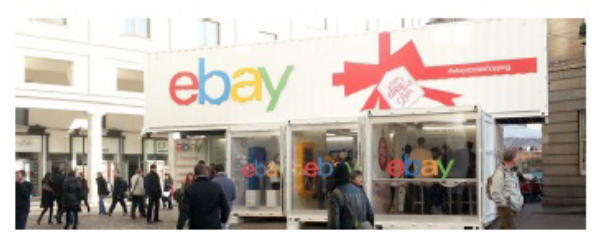




\section{Ocula ${ }^{16}$}

Semiotics of Economic Discourse | Semiotica del discorso economico

Tiziana Barone| Controtendenza del retail nella crisi del nuovo millennio

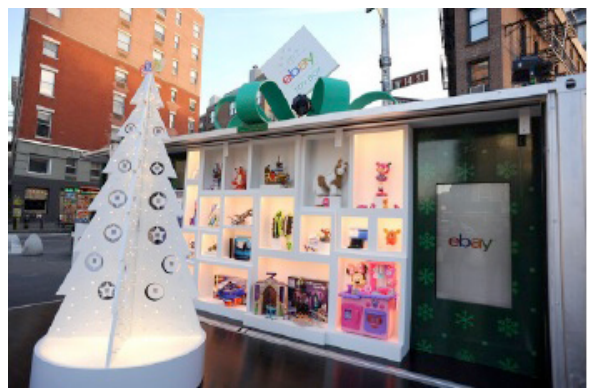

Figura 20.

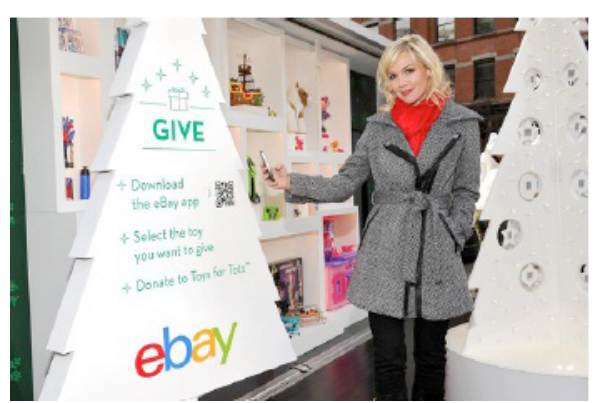

Figura 22.

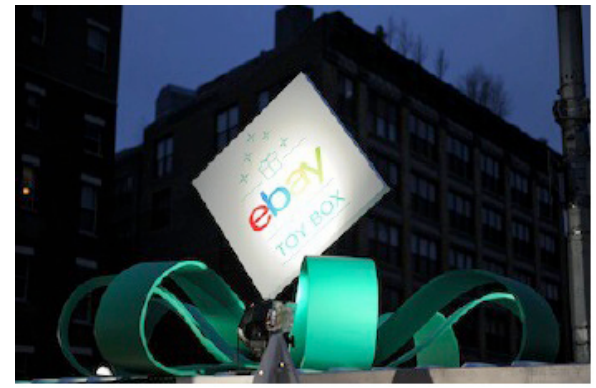

Figura 21.

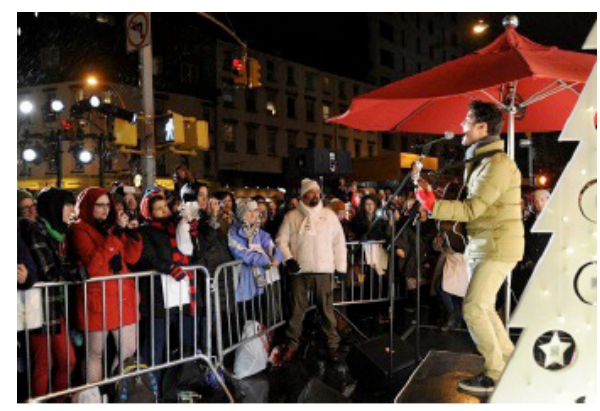

Figura 23.

\section{Bibliografia}

Appadurai, A.

1996 Modernity at large: Cultural dimentions of globalization, University of Minnesota Press, Minneapolis-London (tra. it. Modernità in polvere, Meltemi, Roma, 2001)

Barthes, R.

1970 L'Empire des signes, Skira, Genève, (L'impero dei segni, trad. it Marco Vallora, Einaudi, Torino 1984, 1992.)

Beck, U.

1997 Che cos'è la globalizzazione. Rischi e prospettive della società planetaria, Carocci, Roma

Bolter, JD; Grusin, R.

1999 Remediation. Understanding New Media, The MIT Press, Cambridge (MA) (trad. it. Remediation. Competizione e integrazione tra media vecchi e nuovi, Guerini e Associali)

Ceriani, G.

2007 Hot spots e sfere di cristallo. Semiotica della tendenza e ricerca strategica, Franco Angeli Milano

2013 Semiotica del passaggio. Tra rischio, tensione e tendenza, in As interações sensíveis; A. C. Mei Alves de Oliveira (ed.), Editions Estação das Letras e Cores e Editora CPS, Sao Paulo 


\section{Ocula ${ }^{16}$}

Semiotics of Economic Discourse | Semiotica del discorso economico

Tiziana Barone| Controtendenza del retail nella crisi del nuovo millennio

Fabbris, G.

2003 Il consumatore postmoderno, Franco Angeli Milano

Floch, J. M.

1990 Sémiotique, marketing et communication, PUF, Paris (trad. it. Semiotica, marketing e comunicazione, Franco Angeli, Milano, 1997)

1995 Identités Visuelles, PUF, Paris (trad.it Identità Visive, Franco Angeli, Milano, 1997)

2006 Bricolage. Lettere ai semiologi della terra ferma, a cura di M. Agnello, G, Marrone, Meltemi Roma

Giannitrapani, A.

2013 Introduzione alla semiotica dello spazio, Carocci

Hammad, M.

$2001 \quad$ Lire l'espace, comprendre l'architecture, PUF, Paris (trad. it. Leggere lo spazio comprendere l'architettura, Meltemi, Roma 2003)

Landowski, E. e Marrone G.

2002 (a cura di) La società degli oggetti, Meltemi editore

Landowski, E.

1989 La société réflechie, Seuil, Paris (trad. it. La società riflessa, Meltemi, Roma, 1999)

Latour, B.

1992 Where are the missing masses? The sociology of a few mundane artefact, in Bijiker, Law (1992), (trad. it. Dove sono le masse mancanti? Sociologia di alcuni oggetti di uso comune, in Mattozzi, 2006a)

Lotman, J.M.

1980 Testo e contesto. Semiotica dell'arte e della cultura, Laterza, Roma-Bari

Mangano, D.

2008 Semiotica e design, Carocci

Marrone, G.

2001 Corpi sociali, Piccola Biblioteca Einaudi

2007 Il discorso di marca, Ed. Laterza

2013 Figure di città - Spazi urbani e discorsi sociali, Mimesis

Pastore, A. e Vernuccio, M.

2012 Impresa e comunicazione, Apogeo

Pezzini, I. e Spaziante L.

2014 (a cura di) Corpi mediali, Edizioni ETS

Sloman, J.

2007 Elementi di economia, Il Mulino

Turri, M.G.

2013 La relazione fra economia e finanza. Di cosa parliamo, Lo Spazio filosofico

Zunzuneghi, S.

2011 Metamorfosi dello sguardo. Museo e semiotica, Nuova Cultura 